\title{
Correction to: Exploitation of optical and SAR amplitude imagery for landslide identification: a case study from Sikkim, Northeast India
}

\author{
Thota Sivasankar · Swakangkha Ghosh • \\ Mayank Joshi
}

Published online: 28 July 2020

(C) Springer Nature Switzerland AG 2021

\section{Correction to: Environ Monit Assess (2021) 193:386 https://doi.org/10.1007/s10661-021-09119-6}

The original version of this article unfortunately contained a mistake. The sub-section under Satellite datasets and pre-processing section contained two subsections namely, 1) Optical data and pre-processing, and 2) SAR data and pre-processing. These two sections contained misplaced texts. The second paragraph from the subsection, Optical data and pre-processing contained texts from the subsection SAR data and pre-processing and vice versa. The misplaced texts have been replaced below.

\section{Optical data and pre-processing}

Sentinel-2 mission is a constellation of two earth observation satellites which provides wide-swath, high-resolution,

The original article can be found online at https://doi.org/ 10.1007/s10661-021-09119-6.

T. Sivasankar

NIIT University, Neemrana (Rajasthan), India

\section{S. Ghosh $(\bowtie)$}

Indian Institute of Technology (Indian School of Mines),

Dhanbad, India

e-mail: swakangkhaghosh23@gmail.com

M. Joshi

National Institute of Himalayan Environment and Sustainable Development, Sikkim Regional Center, Gangtok (Sikkim), India multispectral imagery. In the present study, Level-1C product representing Top-of-Atmosphere (TOA) reflectance images and ortho-rectified images is considered for the analysis (Gascon et al., 2014). This product contains 13 spectral bands at various spatial resolutions, out of which, bands 4, 6, and 3 are at 10-m, 20-m, and 60-m spatial resolutions respectively (Van der Meer et al., 2014). However, the present study uses only green $(560 \mathrm{~nm})$, red $(665 \mathrm{~nm})$, near-infrared (NIR, $865 \mathrm{~nm}$ ), and short-wave infrared (SWIR, $2190 \mathrm{~nm}$ ) bands. In order to assess the potential of Sentinel-2 data for automatic landslide detection, the cloudfree imagery acquired shortly before and after the landslide on 13 August 2016 are used in this study (see Fig. 3). These bands are resampled to 20-m pixel spacing for further analysis. In addition, the pre- and post-landslide imagery are co-registered, using pre-landslide data as master and postlandslide data as slave, to effectively analyze the underlying changes which occurred due to the landslide.

It is a known fact that landslides in vegetated areas often cause reduction of standing vegetation on slopes and results in bare-earth exposure. Thus, this study considers "percentage red reflectance change", calculated using Eq. 1 from pre and post event data acquired from the band at $665 \mathrm{~nm}$.

$\%$ RedChange $=\frac{R 665 \mathrm{~nm}_{\text {post }}-R 665 \mathrm{~nm}_{\text {pre }}}{R 665 \mathrm{~nm}_{\text {pre }}} \times 100$ 
Besides the percentage red reflectance change, normalized multiband drought index (NMDI) is also employed in this study. Wang and Qu (2007) developed NMDI for monitoring soil and vegetation moisture from Moderate resolution Imaging Spectroradiometer (MODIS) imagery. Later, it was modified by Fayne et al. (2019) focusing on similar sensitivities of infrared (IR) and SWIR bands on the Landsat-8 Operational Land Imager (OLI) sensor. Due to its sensitivity towards vegetation as well as changes in soil moisture over bare-earth and sparse vegetation, modified NMDI (mNMDI) is calculated from the corresponding bands of Sentinel-2 data using the Eq. 2 .

$m N M D I=\frac{R 865 n m-R 2190 n m}{R 865 n m+R 2190 n m}$

\section{SAR data and pre-processing}

The C-band (center frequency of $5.405 \mathrm{GHz}$ ) Level-1 GRD Ground Range Detected (GRD) Sentinel-1 SAR images acquired in Interferometric Wide Swath (IW) mode during pre- and post-landslide event are used in this study. Sentinel-1 mission is a constellation of two satellites i.e., Sentinel-1A and 1B, that provides data with a spatial resolution (range $\times$ azimuth) of $5 \times 20 \mathrm{~m}$ with $250 \mathrm{~km}$ swath width in IW mode. These satellites acquire data at temporal resolution of 12 days or 6 days depending on the availability of Sentinel-1A and 1B imagery with a look angle ranging from $29.1^{\circ}$ to $46^{\circ}$, in both VV and VH polarization (Malenovsky et al., 2012).
In the present study, SAR imagery acquired in the VV polarization are employed to exploit its potentials for landslide identification. $\mathrm{VH}$ polarization was not operationally available during the initial days of this mission. The pre-processed Sentinel-1 SAR datasets provided by a cloud-based platform named Google Earth Engine (GEE), has been used in this study. The pre-processing in GEE involves: 1) applying orbit file, to update orbit metadata with a restituted orbit file, 2) implementing thermal noise removal which reduces noise effects in the inter-sub-swath texture, 3) applying radiometric calibration which generates a sigma naught backscatter in decibel $(\mathrm{dB})$ from DN imagery using sensor calibration parameters, and 4) terrain correction which transforms the slant-range to groundrange using SRTM $30 \mathrm{~m}$ DEM or the ASTER DEM for high latitudes (greater than $60^{\circ}$ or less than $-60^{\circ}$ ). In addition to these, the datasets are pre-processed with GRD border noise removal from January 2018 (GEE, 2020). These images are further pre-processed with speckle filtering (i.e., $7 \times 7$ adaptive Sigma Lee filter) (Lee et al., 2008) in the Sentinel-1 Toolbox to reduce inherent granular noise. The specifications for GRD Sentinel-1 SAR imagery used in this study are given in Table 1.

Publisher's Note Springer Nature remains neutral with regard to jurisdictional claims in published maps and institutional affiliations. 